\title{
Acute histoplasmosis in immunocompetent travelers: a systematic review of literature
}

\author{
Silvia Staffolani ${ }^{1,2^{*}}$ (D), Dora Buonfrate ${ }^{1 \dagger}$, Andrea Angheben ${ }^{1 \dagger}$, Federico Gobbi ${ }^{1}$, Giovanni Giorli, Massimo Guerriero ${ }^{3}$,
} Zeno Bisoffi ${ }^{1,4}$ and Francesco Barchiesi ${ }^{5}$

\begin{abstract}
Background: Histoplasmosis is a fungal infection highly endemic in the American continent. The disease can be severe in immunocompromised subjects. In immunocompetent subjects the clinical manifestations are variable. Aim of this work was to review the cases of acute histoplasmosis in immunocompetent travelers reported in literature.

Methods: A systematic review of literature was conducted. Electronic search was performed in Pubmed and LILACS. Two reviewers independently extracted data on demographic, clinical and radiological features, and treatment. Cases were classified according to Wheat's definitions.

Results: Seventy-one studies were included in the analysis, comprising a total of 814 patients. Twenty-one patients diagnosed at the Centre of Tropical Diseases, Negrar (VR), Italy were also included. The most common travel destination was Central America (168 people, 29.8\%); the most common way of exposure to histoplasma was the exploration of caves and/or contact with bat guano (349 people, 60.9\%). The multivariate logistic regression model showed association between the development of disseminated histoplasmosis (DH) and activities that involved the exploration of caves and/or the contact with bats' guano (adjusted OR: 34.20 95\% Cl: 5.29 to 220.93) or other outdoor activities (adjusted OR: 4.61 95\% Cl: 1.09 to 19.56). No significant difference in the attack rate between countries of destination was observed (p-value: 0.8906, Kruskal-Wallis test).

Conclusions: Histoplasmosis often causes no or mild symptoms in immunocompetent individuals, although a severe syndrome may occur. The infection can mimic other diseases, and the epidemiological risk of exposure is an important clue to raise the index of suspicion.
\end{abstract}

Keywords: Histoplasma, Histoplasmosis, Acute, Travel, Immunocompetent

\section{Background}

Histoplasmosis is a fungal infection acquired by inhalation of Histoplasma capsulatum var. capsulatum microconidia. This fungus is endemic in the American continent (North, Central and South America). Cases have also been described in Africa, Asia, and Europe [1].

Histoplasmosis is both a disease originating from soil (infections often occur after human disruption of the soil

\footnotetext{
* Correspondence: silvia.staffolani@gmail.com

${ }^{\dagger}$ Dora Buonfrate and Andrea Angheben contributed equally to this work.

${ }^{1}$ Centro per le Malattie Tropicali, IRCCS Istituto di Ricovero e Cura a Carattere Scientifico, Ospedale Sacro Cuore - Don Calabria, Via Don Sempreboni 5,

37024 Verona, Negrar, Italy

${ }^{2}$ SOD Malattie Infettive emergenti e degli immunodepressi, Azienda

Ospedaliero Universitaria, Ospedali Riuniti di Ancona, Via Conca Torrette,

Ancona, Italy

Full list of author information is available at the end of the article
}

that aerosolizes the hyphae and conidia of the organism originating from the earth enriched with birds' excreta) and a sapronosis (originating from bats) [2,3]. With rare exceptions, this fungus infects.

its host via the respiratory route: microconidia are inhalated and deposited in the terminal bronchioles and alveoli of the lung.

Clinical manifestations of histoplasmosis depend on the size of the inoculum and the patient's immunological status/underlying conditions. Low level of exposure in the healthy host typically leads to an asymptomatic infection, while the acute syndrome usually follows a heavy inoculum [4]. In the latter case, chest radiographs usually show diffuse interstitial or reticulonodular infiltrates, sometimes associated with hilar or mediastinal lymphadenopathy [4]. 
Systemic symptoms include fever with possible respiratory impairment, sweats, weight loss, headache and gastrointestinal complaints. A subacute pulmonary syndrome is also possible in case of low inoculum [4]. The most frequent symptoms are cough, fever, malaise and fatigue, typically occurring over weeks to months before seeking medical advice [4]. Solitary lung nodules (histoplasmomas) or multiple pulmonary nodules may develop, characterized by indefinite persistence of foci of infection.

Calcification is usually found in the center of a histoplasmoma or in concentric rings and is generally diagnostic, although it may require years to develop and is not always present.

Pulmonary nodules may slowly enlarge and even cavitate [5]; moreover, they are usually identified as incidental findings on chest radiographs or CT scans, and differential diagnosis includes sarcoidosis, tuberculosis (TB) and malignancies [6-8]. Chronic pulmonary histoplasmosis can be observed in individuals with underlying lung conditions, and it is defined as failure to clear $H$. capsulatum infection [4].

Symptoms and signs include cough, low-grade fever, night sweats and weight loss. Chest radiographs show interstitial or consolidative infiltrates associated with cavitation or fluid-filled emphysematous bullae [4]. This kind of pulmonary syndrome doesn't resolve spontaneously, leading to gradual progression and destruction of lung parenchyma. Mediastinitis, broncholitiasis, pericarditis and a rheumatologic syndromes have been described, too. The disease is particularly relevant for immunosuppressed subjects, who are at risk of developing the disseminated form, which is often fatal if not promptly recognized [4].

On the other hand, the infection is usually mild and self-limiting in immunocompetent people [4], although, apparently in rare cases, the disease can progress to a severe form with high morbidity even in those individuals [9-12].

\section{Objectives}

Aim of our work was to review the literature on acute histoplasmosis in immunocompetent travelers, describing the main characteristics of the infection in this subgroup of patients. Secondary objectives were to investigate the characteristics associated to the development of disseminated histoplasmosis in travelers, and to estimate the attack rate in different areas of the world.

\section{Methods}

We conducted a systematic review of case reports and case series available in literature. We analyzed the epidemiological and clinical features, diagnostic work-up, treatment, and outcome of cases of acute histoplasmosis in immunocompetent patients, with history of recent travel ( $\leq$ 3 months) in an endemic area.
Electronic search was conducted in PubMed and LILACS on 31st December 2016, and all papers published up to that date were included. We included full texts written in English, Italian, French, German, Spanish, Portuguese.

Additional cases were sought from the reference list of included papers and of non-included reviews. Moreover, grey literature was sought through contact with authors. Two authors, SS and AA, independently screened the list of papers generated from the electronic search, using EndNote program, version 6, 2012 Thomson Reuters.

SS and AA extracted the data on the basis of inclusion/ exclusion criteria and case definitions (reported below), and entered all information in a database created with Microsoft Office Excel 2007. Data collected were: age, sex, country of exposure, activities done in the country, attack rate and development of disseminated disease. The Excel data base is available as supplementary material (Additional file 1).

In case of discrepancies in the process of inclusion of papers/data extraction, a consensus was reached through discussion or involvement of a third reviewer (FG).

\section{Inclusion criteria}

-Case reports or case series on acute histoplasmosis.

-Patients with history of travel in an endemic area (including travels from an area of no/low endemicity to an area of high endemicity within a country) $\leq 3$ months before the onset of symptoms.

-Immunocompetence status.

\section{Exclusion criteria}

-Reviews of literature.

-Cases describing patients presenting with radiologic findings compatible with histoplasmosis (i.e. pulmonary nodules) but no apparent temporal relationship with travel.

-Cases native from an endemic area without history of travel to an area with higher endemicity.

-Non-immunocompetence status at the time of the travel, at the onset of symptoms or at the time of diagnosis of histoplasmosis.

-Occupational exposure in native area.

-Cases of reactivation of histoplasmosis acquired in the past.

-Cases of histoplasmosis due to Histoplasma capsulatum var. duboisii.

Case definition (adapted from wheat [13] and EORTC/MSG criteria [14])

Proven

Illness consistent with acute histoplasmosis in a patient who had recently ( $\leq 3$ months) travelled to an endemic area and one of the following: 
-Positive culture from a specimen obtained from the affected site or tissue.

-Histopathologic or direct microscopic demonstration of yeast intracellular forms or yeasts in tissue macrophages.

\section{Probable}

-Host factor: recent travel ( $\leq 3$ months) in area endemic for histoplasmosis.

-Clinical picture and/or radiological findings consistent with acute histoplasmosis.

-Mycological evidence (histoplasmin test positivity and/or positivity of serology and/or positivity of urine-serum Histoplasma antigen).

\section{Possible}

-Host factor: recent travel ( $\leq 3$ months) in area endemic for histoplasmosis.

-Clinical picture and/or radiological findings consistent with acute histoplasmosis.

-No mycological evidence nor serologic evidence but:

Belonging to a histoplasmosis cluster and/or efficacy of oral ex juvantibus itraconazole therapy (resolution of symptoms and normalization of radiological findings).

\section{Activities at risk for developing histoplasmosis}

Contact with bats excreta and/or visit to a cave.

Outdoor activities: forest excursions/trekking, camping or other activities not involving visit to caves or direct contact with bats' excreta.

Activities involving contact with chickens or other non-mammal birds.

All activities that put the subject at risk of inhalating Histoplasma microconidia: building construction, renovation, excavation (biologists, building contractors, construction workers, soldiers, volunteers involved in churches or other buildings renovation).

\section{Syndromic classification (according to wheat [13])}

Asymptomatic (As): asymptomatic case identified through laboratory screening within a histoplasmosis cluster.

Acute pulmonary histoplasmosis (AP): febrile illness with respiratory symptoms and/or mediastinal involvement and/or accompanying constitutional symptoms and/or gastrointestinal or cutaneous involvement when they are not the main manifestation.

Rheumathologic $(\mathrm{R})$ : a case with arthralgia or arthritis as unique symptom.

Disseminated Histoplasmosis (DH): febrile illness with demonstration of bone marrow involvement (leucopenia, thrombocytopenia, anemia) AND/OR disseminated monocytic-macrophage involvement (hepatosplenomegaly AND/OR increased liver enzymes), with or without respiratory symptoms.

\section{Statistical analysis}

Descriptive statistics were used to analyze the characteristics of the entire cohort. Categorical variables were reported as frequencies and proportions, while quantitative variables were presented as median (interquartile range, IQR). Associations between covariates were explored through univariate logistic models and non-parametric Kruskal-Wallis rank sum test. Lastly, we fitted a multivariate logistic regression model to assess the impact of all covariates on the probability of contracting the disseminated disease. All statistical analyses were conducted using $R$, version 3.3.3 [15].

\section{Results}

The study selection process (PRISMA flow chart) is shown in Fig. 1. The studies included in the analysis through electronic search were 71 [8, 16-85], (Table 1) comprising a total of 814 patients (see Fig. 2 for the timeline distribution of studies and cases respectively). In the analysis were also included 21 patients diagnosed at the Centre of Tropical Diseases (CTD) of Negrar, Verona, Italy during the decade 2005-2015 (Staffolani S, Buonfrate D, Farina C, Gulletta M, Gobbi F, Angheben A, manuscript in preparation), for a total of 835 patients. Figure 3 shows the countries of presumable exposure to the infection.

Regarding the cases diagnosed at the Centre of Tropical Diseases, Negrar (VR), briefly, we found 21 cases of acute histoplasmosis in immunocompetent subjects, 17 of them affecting members of a scientific expedition to Ecuador. The other 4 patients did not belong to a cluster and traveled to Panama, Bolivia, Mexico, Cuba respectively. Seventeen out of 21 patients were male $(81 \%)$. The mean age was 38.5 years for the Ecuador cluster, 46.7 years for the other cases. They were infected through contact with bat excreta, inhalation of contaminated soil, outdoor activities. All cases were classified as possible histoplasmosis, but two probable for positive serology and two proven cases based on histology. The main symptoms were fever $(12 / 21,57 \%)$, respiratory $(12 /$ $21,57 \%)$ and gastrointestinal $(9 / 21,43 \%)$ symptoms. Seven patients (33\%) were admitted to the hospital and four of them underwent invasive diagnostic procedures (bronchoscopy, biopsy of other tissues). Eleven out of 17 (65\%) patients of the Ecuador cluster and 4/4 (100\%) patients not belonging to it had abnormal blood exams. Eleven patients $(65 \%, 7 / 17(41 \%)$ in the cluster and $4 / 4$ (100\%) not belonging to the Ecuador cluster) received oral itraconazole. Nine patients $(39.1 \%)$ had persistent lung nodules at the 12-month radiological follow up.

Regarding all selected cases of the present review, most patients $(488 / 835,59 \%)$ belonged to a cluster.

There were 45 clusters described by 36 authors, plus 2 clusters diagnosed at CTD. The cluster comprising the 


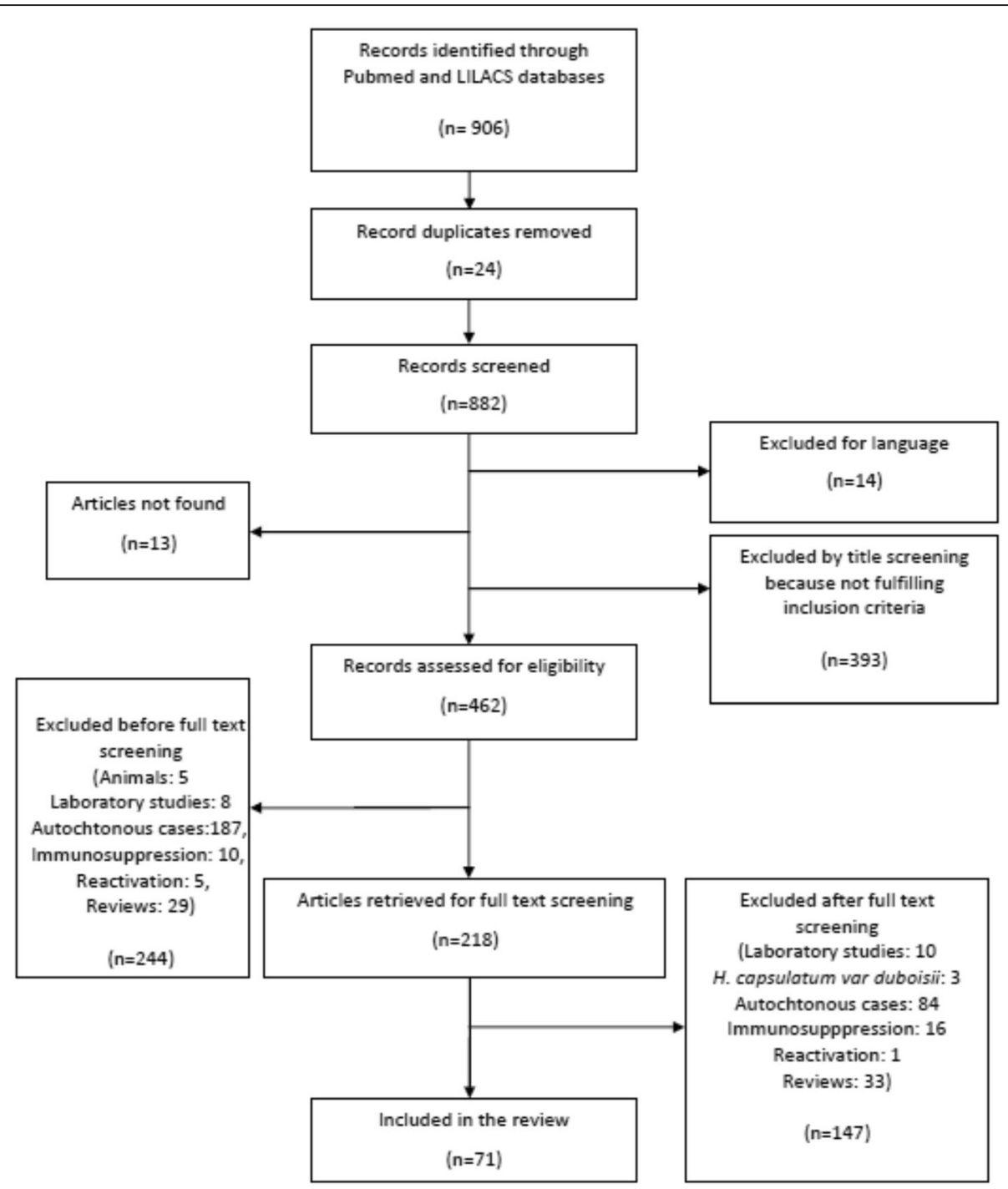

Fig. 1 PRISMA flow chart for study selection

largest number of individuals $(n=262)$ was described by Morgan [35].

While we considered all 835 patients for the description of main characteristics of cases, the "Acapulco cluster" [35] was removed from our dataset for univariate and multivariate analyses, as it did not contain information regarding age or the state of disseminated disease. Table 2 displays a summary of the characteristics of the cohort of the 573 immunocompetent travelers considered for univariate and multivariate analyses.

\section{Clinical-syndromic classification}

According to the above-mentioned definitions, we found 32 proven, 695 probable and 108 possible histoplasmosis cases.

Most patients $(605 / 724,84 \%)$ had acute pulmonary histoplasmosis (AP). Of them, 111 (18\%) had also rheumatologic manifestations and 26 (4\%) mediastinal involvement. In this group we also included patients with miliary pulmonary lesions if they had only respiratory symptoms with no signs of dissemination. Fifty-six (8\%) cases were classified as Disseminated Histoplasmosis (DH), 65 (9\%) patients were asymptomatic (A), 9 (1\%) patients had only rheumatologic (R) manifestations.

\section{Symptoms}

This information was available for 789/835 (94.5\%) subjects, of whom 724 reported symptoms. As it is shown in Fig.4, the most frequent symptoms were fever (659/ $724,91 \%)$, cough $(398,55 \%)$, headache $(377,52 \%)$ and chest pain (299, 41\%). Constitutional symptoms (myalgia and/or sweats, and/or weight loss, and/or anorexia) were reported by 418 (58\%) patients. Patients reporting chills, with or without documented fever, were 91 (13\%). 
Table 1 Studies selected for the systematic review

\begin{tabular}{|c|c|c|c|c|c|c|c|c|c|c|c|c|}
\hline & Reference & $\begin{array}{l}\text { Publication } \\
\text { year }\end{array}$ & $\begin{array}{l}\mathrm{N}^{0} \\
\text { cases }\end{array}$ & $\begin{array}{l}\text { Country of } \\
\text { origin }\end{array}$ & $\begin{array}{l}\text { Visited } \\
\text { continent }\end{array}$ & Visited country & $\begin{array}{l}\text { Reason } \\
\text { of travel }\end{array}$ & Exposure & Cluster & $\begin{array}{l}\text { Syndromic } \\
\text { classification }\end{array}$ & Therapy & $\begin{array}{l}\text { Type of } \\
\text { therapy }\end{array}$ \\
\hline 1 & {$[8]$} & 2015 & 1 & Swiss & SA & Brazil, Argentina & & C & no & AP & yes & itra \\
\hline 2 & [16] & 1988 & 15 & NS & CA & Costa rica & ST & B & yes & AP & no & \\
\hline 3 & [17] & 2007 & 4 & Spain & SA & Ecuador & V & $C, S$ & yes & AP & no & \\
\hline 4 & [18] & 2004 & 1 & France & SA & Colombia & T & B & no & AP & yes & NS \\
\hline 5 & [19] & 2011 & 2 & Italy & SA & Ecuador & & $\mathrm{O}$ & no & $A P, D H$ & 1 yes & Itra \\
\hline 6 & [20] & 1992 & 1 & Germany & SA & Ecuador & $\mathrm{T}$ & $\mathrm{O}$ & no & AP & no & \\
\hline 7 & [21] & 2013 & 1 & Israel & SA, A & $\begin{array}{l}\text { Jordan, Bolivia, Brazil, } \\
\text { Ethiopia, Angola }\end{array}$ & $\mathrm{T}$ & $\mathrm{O}$ & no & $\mathrm{DH}$ & yes & Itra \\
\hline 8 & [22] & 1995 & 2 & France & SA & Guyana & $P$ & 0,5 & no & AP & yes & Excision \\
\hline 9 & [23] & 2011 & 5 & Spain & $A, S A, C A$ & $\begin{array}{l}\text { Angola, Venezuela, } \\
\text { Nicaragua, Ecuador }\end{array}$ & $\begin{array}{l}T, P, S P \\
V\end{array}$ & $\mathrm{~B}, \mathrm{O}$ & no & AP & no & \\
\hline 10 & [24] & 2002 & 14 & Canada & CA & Belize & ST & $\mathrm{O}$ & yes & AP & 1 yes & itra \\
\hline 11 & [25] & 2013 & 1 & USA & SA & Peru & V & $B, S$ & no & $\mathrm{DH}$ & yes & $A m B$, itra \\
\hline 12 & [26] & 1999 & 1 & France & SA & Guyana & $P$ & $\mathrm{O}, \mathrm{S}$ & no & $\mathrm{DH}$ & yes & itra \\
\hline 13 & [27] & 2013 & 12 & $\begin{array}{l}\text { Europe, } \\
\text { Africa }\end{array}$ & A & Uganda & ST & $\mathrm{O}$ & yes & AP & 5 yes & $\begin{array}{l}4 \text { itra, } 1 \\
\text { keto }\end{array}$ \\
\hline .14 & [28] & 1979 & 10 & & A & South Africa & SP & B & yes & $9 \mathrm{AP}, 1 \mathrm{DH}$ & 1 yes & $A m B$ \\
\hline 15 & [29] & 1997 & 1 & France & SA & Guyana & $P$ & $0, \mathrm{~S}$ & no & AP & yes & Itra \\
\hline 16 & [30] & 2006 & 1 & Netherland & A & Ghana & $\mathrm{T}$ & $\mathrm{B}, \mathrm{O}$ & no & AP & no & \\
\hline 17 & [31] & 1957 & 2 & $\begin{array}{l}\text { South } \\
\text { africa }\end{array}$ & A & South Africa & SP & B & yes & AP & no & \\
\hline 18 & [32] & 2012 & 1 & Germany & SA & Costa Rica & $\mathrm{T}$ & $\mathrm{B}, \mathrm{O}$ & no & AP & yes & itra \\
\hline 19 & [33] & 2002 & 6 & Germany & CA & Cuba & $\mathrm{BIO}$ & $B, S$ & yes & $3 \mathrm{AP}, 3 \mathrm{DH}$ & yes & itra \\
\hline 20 & [34] & 2010 & 3 & Japan & & Malaysia & $\mathrm{T}$ & $\mathrm{O}$ & yes & AP & no & \\
\hline 21 & [35] & 2003 & 262 & USA & & Mexico & T & 0,5 & yes & AP & yes & NK \\
\hline 22 & [36] & 2000 & 4 & Italy & $C A, S A$ & $\begin{array}{l}\text { Guatemala, } \\
\text { Dominican republic, } \\
\text { Peru }\end{array}$ & $\mathrm{T}, \mathrm{SP}$ & $B, O, S$ & no & $3 \mathrm{AP}, 1 \mathrm{DH}$ & yes & $\begin{array}{l}\text { 2keto, } 1 \\
\text { itra, } 1 \mathrm{amB}\end{array}$ \\
\hline 23 & [37] & 2005 & 1 & Italy & CA & Nicaragua & $P$ & $0, \mathrm{~S}$ & no & AP & yes & itra \\
\hline 24 & [38] & 2003 & 3 & Spain & CA & Nicaragua & $\mathrm{T}$ & $\mathrm{B}, \mathrm{O}$ & yes & AP & 1 yes & itra \\
\hline 25 & [39] & 1988 & 11 & USA & USA & lowa & $\mathrm{T}$ & $\mathrm{O}, \mathrm{S}$ & yes & AP & no & \\
\hline 26 & {$[40]$} & 2005 & 9 & Spain & SA & Guatemala & V & S & yes & AP & no & \\
\hline 27 & [41] & 2000 & 7 & Spain & CA, SA & $\begin{array}{l}\text { Dominican Republic, } \\
\text { Nicaragua, Colombia, } \\
\text { Peru, Guatemala }\end{array}$ & $T, V$ & $B, O, S$ & no & $1 \mathrm{AP}, 8 \mathrm{DH}$ & yes & itra \\
\hline 28 & {$[42]$} & 1962 & 3 & Europe & A & South Africa & SP & B & yes & $\mathrm{DH}$ & yes & $A m B$ \\
\hline 29 & [43] & 1990 & 1 & France & SA & Guyana & $\mathrm{T}$ & $\mathrm{O}$ & no & AP & no & \\
\hline 30 & [44] & 1981 & 69 & USA & NA & South Carolina & $\mathrm{Cl}$ & C & yes & AP & no & \\
\hline 31 & [45] & 1975 & 6 & Canada & CA & Puerto Rico & ST, TEA & $\mathrm{B}, \mathrm{O}$ & yes & AP & no & \\
\hline 32 & {$[46]$} & 1979 & 1 & Jamaica & CA & Caribbean & TEA & B & no & AP & no & \\
\hline 33 & {$[47]$} & 1996 & 1 & Germany & CA & & $\mathrm{T}$ & $\mathrm{O}$ & no & AP & no & \\
\hline 34 & [48] & 2008 & 3 & Austria & & Mexico & $\mathrm{T}$ & B & no & $\mathrm{DH}$ & yes & itra \\
\hline 35 & [49] & 2008 & 3 & Germany & CA & Antille & $\mathrm{BIO}$ & $B, S$ & yes & $2 \mathrm{DH}, 1 \mathrm{AP}$ & yes & itra \\
\hline 36 & {$[50]$} & 2012 & 4 & Poland & SA & Ecuador & T & B & yes & $\mathrm{DH}$ & 2 yes & keto \\
\hline 37 & [51] & 2011 & 2 & France & CA & Costa Rica & $\mathrm{T}$ & $\mathrm{O}$ & yes & AP & 1 yes & 1 itra \\
\hline 38 & {$[52]$} & 2003 & 1 & Germany & & Mexico, Brazil & $\mathrm{T}$ & O & no & AP & no & \\
\hline 39 & {$[53]$} & 1979 & 27 & USA & USA & North-Centre Florida & V & $\mathrm{B}, \mathrm{O}, \mathrm{S}$ & yes & $\begin{array}{l}8 \mathrm{As}, 2 \mathrm{DH} \\
17 \mathrm{AP}\end{array}$ & 2 yes & $2 \mathrm{AmB}$ \\
\hline
\end{tabular}


Table 1 Studies selected for the systematic review (Continued)

\begin{tabular}{|c|c|c|c|c|c|c|c|c|c|c|c|c|}
\hline & Reference & $\begin{array}{l}\text { Publication } \\
\text { year }\end{array}$ & $\begin{array}{l}\mathrm{N}^{\circ} \\
\text { cases }\end{array}$ & $\begin{array}{l}\text { Country of } \\
\text { origin }\end{array}$ & $\begin{array}{l}\text { Visited } \\
\text { continent }\end{array}$ & Visited country & $\begin{array}{l}\text { Reason } \\
\text { of travel }\end{array}$ & Exposure & Cluster & $\begin{array}{l}\text { Syndromic } \\
\text { classification }\end{array}$ & Therapy & $\begin{array}{l}\text { Type of } \\
\text { therapy }\end{array}$ \\
\hline 40 & {$[54]$} & 2004 & 10 & USA & CA & Costa Rica & $T$ & B & yes & AP & no & \\
\hline 41 & [55] & 2013 & 1 & Canada & CA & Costa Rica & & $\mathrm{B}, \mathrm{O}, \mathrm{S}$ & no & $\mathrm{DH}$ & yes & AmB, itra \\
\hline 42 & [56] & 2005 & 2 & France & SA & Venezuela & $P$ & $\mathrm{O}, \mathrm{S}$ & no & AP & yes & itra \\
\hline 43 & [57] & 1991 & 1 & France & SA & Guatemala & T & $\mathrm{O}, \mathrm{S}$ & no & AP & yes & keto \\
\hline 44 & [58] & 2014 & 1 & Germany & SA & Ecuador & T & $\mathrm{O}$ & no & AP & yes & itra \\
\hline 45 & [59] & 1957 & 64 & Europe, NS & A & South Africa & $S T, S P$ & B & yes & AP, 5 As & no & \\
\hline 46 & [60] & 1997 & 4 & Italy & SA & Peru & SP & $B, C$ & yes & $\mathrm{DH}$ & yes & keto \\
\hline 47 & [61] & 1995 & 24 & $\begin{array}{l}\text { Europe, } \\
\text { SEA }\end{array}$ & $\mathrm{AU}$ & New Caledonia & SP & B & yes & $\mathrm{AP}, 3 \mathrm{As}$ & 21 yes & $\begin{array}{l}\text { Keto, itra, } \\
\mathrm{AmB}\end{array}$ \\
\hline 48 & [62] & 2009 & 5 & Spain & CA, SA & $\begin{array}{l}\text { Peru, Costa Rica, El } \\
\text { Salvador, Panama, } \\
\text { Ecuador }\end{array}$ & $\begin{array}{l}\mathrm{T}, \mathrm{BIO}, \mathrm{V}, \\
\mathrm{P}\end{array}$ & $\mathrm{B}, \mathrm{C}, \mathrm{O}, \mathrm{S}$ & no & $3 \mathrm{AP}, 2 \mathrm{DH}$ & $\begin{array}{l}\text { Yes } 1 \\
\text { pulmonary } \\
\text { and } 2 \mathrm{DH}\end{array}$ & itra \\
\hline 49 & [63] & 2001 & 1 & Spain & SA & Peru & T & $\mathrm{B}, \mathrm{O}$ & no & AP & yes & itra \\
\hline 50 & [64] & 2014 & 4 & Brazil & SA & Brazil & $\mathrm{BIO}$ & B & yes & AP & 2 yes & itra \\
\hline 51 & [65] & 1986 & 6 & USA & USA & Florida & $S T, P$ & $B, S$ & yes & AP & 4 yes & 2Amb,2keto \\
\hline 52 & [66] & 2006 & 1 & USA & CA & Guatemala & T & $\mathrm{O}$ & no & $\mathrm{R}$ & yes & itra \\
\hline 53 & [67] & 2003 & 13 & France & CA & Martinica & $\begin{array}{l}\text { Trekking } \\
\text { trip }\end{array}$ & 0,5 & yes & $11 \mathrm{DH}, 2 \mathrm{AP}$ & yes & itra \\
\hline 54 & [68] & 2009 & 1 & Germany & $C A$ & Antille & $\mathrm{BIO}$ & $B, S$ & no & AP & yes & NS \\
\hline 55 & [69] & 2015 & 23 & Israel & $\begin{array}{l}\text { USA, CA, } \\
\text { SA }\end{array}$ & $\begin{array}{l}\text { Guatemala, Costa } \\
\text { Rica Peru, Mexico, } \\
\text { Bolivia, Indiana, } \\
\text { Dominican Republic }\end{array}$ & NS & $\mathrm{B}, \mathrm{O}$ & no & $14 \mathrm{AP}, 9 \mathrm{As}$ & Not known & \\
\hline 56 & [70] & 2011 & 3 & France & $C A$ & Cuba & SP & B & yes & $1 \mathrm{DH}, 2 \mathrm{AP}$ & yes & itra \\
\hline 57 & [71] & 2007 & 1 & Japan & SA & Bolivia & $P$ & $\mathrm{O}, \mathrm{S}$ & no & AP & yes & fluco \\
\hline 58 & [72] & 1992 & 1 & Italy & A & & $P$ & $0, \mathrm{~S}$ & no & AP & yes & $A m B$ \\
\hline 59 & [73] & 2000 & 12 & Spain & $C A, S A$ & $\begin{array}{l}\text { Guatemala, } \\
\text { Honduras, Nicaragua, } \\
\text { Dominican Republic, } \\
\text { Peru }\end{array}$ & $\mathrm{V}, \mathrm{SP}, \mathrm{T}$ & $\mathrm{B}, \mathrm{O}, \mathrm{S}$ & yes & $7 \mathrm{AP}, 5 \mathrm{As}$ & 8 yes & itra \\
\hline 60 & [74] & 1999 & 11 & USA & SA & Ecuador & ST & B & yes & $\begin{array}{l}1 \mathrm{DH}, 8 \mathrm{AP} \text {, } \\
2 \mathrm{not} \\
\text { possible }\end{array}$ & 4 yes & $\begin{array}{l}1 \text { itra, } 3 \\
\text { systemic } \\
\text { azoles }\end{array}$ \\
\hline 61 & [75] & 2008 & 20 & USA & CA & El Salvador & V & $0, S$ & yes & AP & Not known & \\
\hline 62 & [76] & 2003 & 14 & USA & $C A$ & Nicaragua & ST & B & yes & $\begin{array}{l}4 \mathrm{DH}, 8 \mathrm{AP}, \\
2 \mathrm{As}\end{array}$ & yes & itra \\
\hline 63 & [77] & 1999 & 6 & Chile & SA & Ecuador & T & $\mathrm{C}, \mathrm{O}$ & yes & AP & yes & itra \\
\hline 64 & [78] & 1966 & 2 & USA & USA & Missouri, Kentucky & T & C & no & AP & 1 yes & $A m B$ \\
\hline 65 & [79] & 2015 & 1 & USA & USA & Southwest & T & $\mathrm{O}, \mathrm{S}$ & no & AP & yes & itra \\
\hline 66 & [80] & 2010 & 4 & Brasil & SA & Brasil & SP & B & yes & $2 \mathrm{AP}, 2 \mathrm{DH}$ & yes & \\
\hline 67 & [81] & 2012 & 5 & Argentina & SA & Argentina & $\mathrm{O}$ & C & Yes & AP & No & \\
\hline 68 & [82] & 2002 & 1 & Chile & SA & Peru & SP & B & No & AP & Yes & \\
\hline 69 & [83] & 2001 & 31 & Venezuela & SA & Venezuela & SP & B & Yes & AP & No & \\
\hline 70 & [84] & 1981 & 33 & colombia & SA & colombia & SP & B & yes & AP & no & \\
\hline 71 & [85] & 1999 & 1 & Taiwan & SEA & Indonesia & $P$ & $\mathrm{O}$ & no & $\mathrm{DH}$ & yes & $A m B$ \\
\hline $\begin{array}{l}\text { CTD } \\
\text { Negrar }\end{array}$ & & 2016 & 21 & Italy & $S A, C A$ & $\begin{array}{l}\text { Ecuador, Bolivia, } \\
\text { Mexico, Cuba }\end{array}$ & $T, S P, P$ & $\mathrm{~B}, \mathrm{O}, \mathrm{S}$ & $\begin{array}{l}16 \text { yes, } \\
5 \text { no }\end{array}$ & $1 \mathrm{DH}, 20 \mathrm{AP}$ & 11 yes & itra \\
\hline
\end{tabular}

NS not specified, USA United States of America, NA North America, CA Central America, SA South America, A Africa, AU Australia, SEA South East Asia, ST student, $V$ Volunteer, $T$ Tourist, $P$ professional, SP Speleologist, BIO Biologist, CI Correctional Institute TEA Teacher, $B$ Cave_bats, $C$ Birds/chickens, $S$ Moved Soil, O Outdoor activities, DH Disseminated Histoplasmosis, AP Acute Pulmonary Histoplasmosis, AmB Amphotericin B, Itra Itraconazole, Fluco Fluconazole, Keto Ketoconazole 


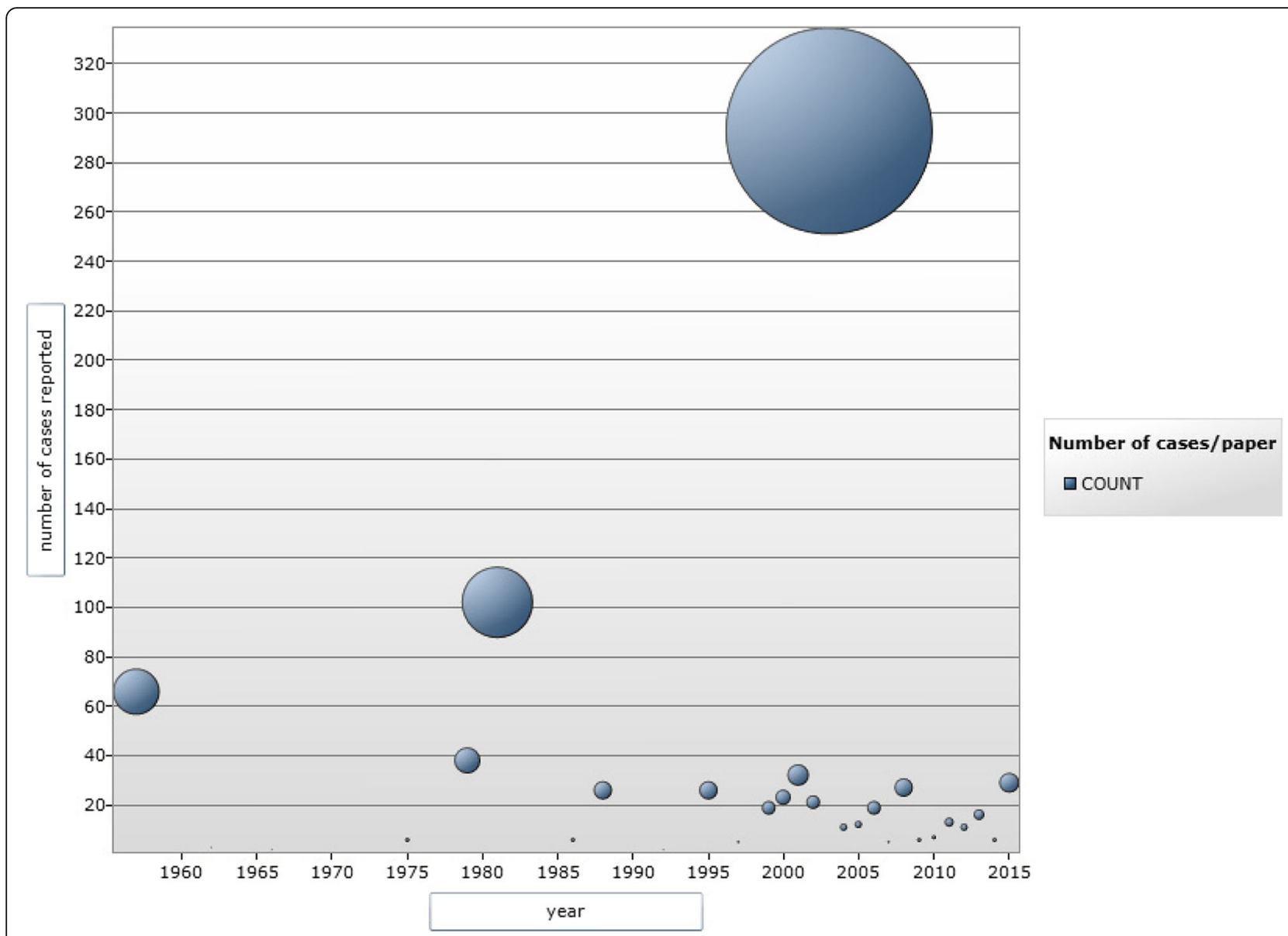

Fig. 2 Timeline distribution of the studies reporting cases of acute histoplasmosis in immunocompetent travelers. Each bubble corresponds to a study, the diameter of the bubble is proportional to the number of cases described in the study, which correspond to the projection of the center of the bubble on the ordinate axis

Gastrointestinal symptoms (158/724, 22\%) comprised diarrhea, nausea, abdominal pain or vomit. Oral lesions (5/724, 0.7\%) were aftous in 3 cases, ulcerative (lingual and tonsillar), and papular (lingual) in one patient each. Skin abnormalities (41/724, 5.5\%) were: diffuse macular rash in $32 / 41$ (78\%) patients, erythema nodosum in 5 $(12 \%)$, while cutaneous ulcers, papules, and splinter hemorrhages were reported in one case each. For a further patient the type of cutaneous lesion was not specified. Symptoms attributed to central nervous system impairment $(14 / 724,2 \%)$ included irritability, insomnia, dizziness, lethargy.

Ophtalmological symptoms $(7 / 724,1 \%)$ were conjunctivitis, ocular pain, photofobia.

\section{Laboratory exams}

This information was available for 212 (25\%) patients. Of them, 90 (42\%) had abnormal laboratory exams (abnormal leucocyte and/or platelet count or anemia, increased inflammatory markers, increased LDH, abnormal liver enzymes values). In particular: 27 (30\%) had abnormal leucocyte and/or platelet count or anemia, 62 (69\%) had increased inflammatory markers, 26 (29\%) had increased LDH, 45 (50\%) had abnormal liver enzymes values.

Information about Histoplasma serology was available for 634 (76\%) patients, of whom 413 (65\%) were positive. All asymptomatic patients included in the analysis were positive to serology, which was performed on the basis of epidemiological suspicion (travel with patients diagnosed with histoplasmosis). Of $213(26 \%)$ patients screened with histoplasmin skin test, 199 (93\%) resulted positive.

\section{Imaging}

Imaging included chest X ray and CT scan. This information was available for $376(45 \%)$ patients. Of them, $250(67 \%)$ showed abnormal imaging. In particular: nodular infiltrates were present in 200 patients (80\%), patches in 16 patients (6\%), miliary pattern in 14 patients (6\%), hylar/mediastinal adenopathies in 49 patients (20\%), pleural effusion in 4 patients (2\%). 


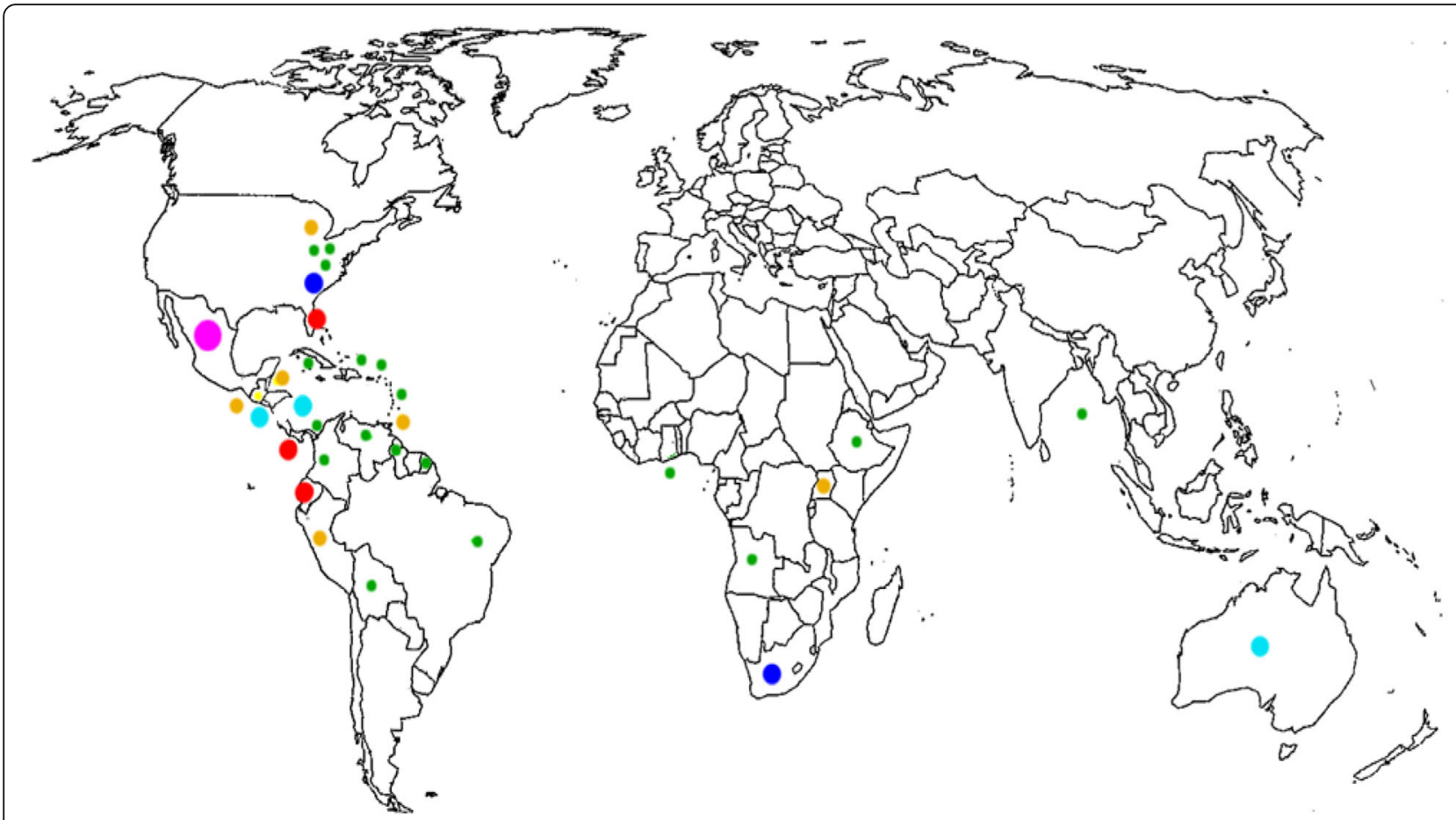

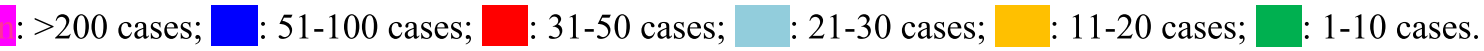

Fig. 3 Distribution of acute histoplasmosis reported cases among immunocompetent travelers

Information about the spleen and liver size was available only for 14 (2\%) patients, of whom 5 (36\%) had hepatosplenomegaly and one had hepatomegaly.

\section{Hospitalization and invasive procedures}

Information about hospitalization was available for $595(71 \%)$ patients, of whom $130(22 \%)$ were admitted to hospital. Information about diagnostic procedures was available for 742 patients (89\%). Of them, 64 patients (9\%) underwent invasive procedures. Usually, obtained specimens were sent for coltural, cytologic and/or histological examination. Fiftyseven out of 62 patients (92\%) had a positive colture for Histoplasma, 9/61 (15\%) had positive cytologic examination (7 of them had a positive hystologic exam, too), 37/63 (59\%) had positive pathology findings (of them 21 had also positive colture and 4 had also a positive cytologic examination).

Fourtyfive patients (70\%) underwent bronchoscopy with bronchoalveolar lavage (BAL), and 21 (47\%) of them underwent lymphonodal biopsy, too. Of all them, 26/44 (59\%) had positive mycologic colture, 8/44 (18\%) had positive cytologic exam and 20/45 (44\%) had positive histologic exam. Eighteen patients had more than one positive result.
Thirteen patients (20\%) were submitted to transthoracic lung biopsy. Seven out of 13 (54\%) coltural exams, $1 / 13$ (8\%) cytologic exams and 9/13 (69\%) histological exams were positive. Four patients had more than one positive exam.

Eight patients (13\%) underwent lung resection. Two out of $8(25 \%)$ coltural and 7/7 (100\%) histological examinations were positive.

Finally, six patients (9\%) underwent biopsies of tissues other than lung (bone marrow 5 patients, skin one patient). Five out of $6(83 \%)$ coltural exams and 5/6 (83\%) histological exams were positive. Four patients had both coltural and histological positive exams.

\section{Treatment}

Information was available for 744 patients $(89 \%)$. Of them, 185 (25\%) received antifungal therapy. In particular: $105(57 \%)$ patients received oral itraconazole, while $21(11 \%)$ received intravenous amphotericin B. Twelve (57\%) of the latter were switched to oral drugs at hospital discharge: one of them was switched initially to oral itraconazole and then to posaconazole, 8 of them were switched to ketoconazole and then to itraconazole, 4 were switched to itraconazole. Twenty-four (13\%) patients were 
Table 2 Baseline Demographic and Travel Characteristics of the Entire Cohort of Immunocompetent Travelers

\begin{tabular}{ll}
\hline Characteristic & Entire Cohort \\
\hline Total & 573 \\
Median age, years (IQR) & $29(23-43)$ \\
Male, N (\%) & $244(64.0)$ \\
Median AR ${ }^{a}, \%$ (IQR) & $90(73.2-100.0)$ \\
Continent of destination, N (\%) & \\
USA-Canada & $117(20.8)$ \\
Central America & $168(29.8)$ \\
South America & $154(27.4)$ \\
Africa & $95(16.9)$ \\
Others ${ }^{b}$ & $29(5.1)$ \\
Activity ${ }^{c}$, N (\%) & \\
Caves/Bat guano & \\
Outdoor activities & $349(60.9)$ \\
Bird_chickens & $198(34.5)$ \\
Inhalation_soil & $105(18.3)$ \\
\hline Abbreviations, AR attack rate, & $104(18.1)$ \\
\hline interquatile range
\end{tabular}

Abbreviations, $A R$ attack rate, $I Q R$ interquartile range

${ }^{\text {a }}$ Calculated as the proportion of infected people in the travel group. We identified 47 travel groups in the cohort

Includes: Asia, Oceania

c Frequencies exceed $100 \%$, as most people did more than one activity during the trip. Caves/Bat's guano: contact with bats excreta and/or visit to a cave. Outdoor activities: forest excursions/trekking, camping or other activities not involving visit to caves or direct contact with bats' excreta. Bird_chickens: activities involving contact with chickens or other non-mammal birds. Inhalation_soil: all activities involving building construction, renovation, excavation (biologists, building contractors, construction workers, soldiers, volunteers involved in churches or other buildings renovation)

treated with systemic azoles other than itraconazole, while 33 (18\%) patients were treated with an unspecified antifungal therapy. A couple of patients with lung nodules underwent excisional surgery and no antifungal therapy.

\section{Outcome}

A detailed information about the outcome was available for 727 (87\%) patients. Most of them (726/727, 99.8\%) recovered. In particular: 688 (95\%) patients had complete clinical and radiological resolution at the last visit, while $38(5 \%)$ patients had clinical recovery and a partial clearance of the radiological findings. One patient with disseminated histoplasmosis and typhoid fever died because of multi-organ failure and hypercalcemia [85].

\section{Univariate and multivariate analyses}

Table 3 reports a summary of the univariate and multivariate analyses conducted on all variables of interest. Results from the univariate analysis showed an association between disseminated histoplasmosis and variables such as age (crude OR: 1.03 95\% CI: 1.01 to 1.05), Central America (crude OR: 13.01 95\% CI: 3.82 to 81.43 ) or
South America (Crude OR: 6.66 95\% CI: 1.84 to 42.72) as a destination, and activities that involved inhalation of contaminated soil (crude OR: 3.13 95\% CI: 1.72 to 5.62 ). However, the multivariate logistic regression model did not confirm any association with destination or age, while a strong association was found with activities that involved the exploration of a cave full of bat's guano (adjusted OR: $34.2095 \%$ CI: 5.29 to 220.93) or other outdoor activities (adjusted OR: 4.61 95\% CI: 1.09 to 19.56). No significant difference in the attack rate between countries of destination was observed ( $p$-value: 0.8906 , Kruskal-Wallis test. Not reported in tables).

\section{Discussion}

We were able to identify in the literature 814 cases of histoplasmosis acquired by immunocompetent travelers, for a total of 835 patients including those diagnosed at CTD. Considering that this occurrence is certainly under-diagnosed and under-reported, we assume that the published cases must be the tip of the iceberg. We hope that our systematic review may contribute to raise awareness of the possible occurrence of this condition in travelers visiting endemic countries/areas. It is true that most cases are clinically not severe, nevertheless most of the subjects presented symptoms of various degree, moreover usually long lasting, often implying otherwise unnecessary, invasive diagnostic procedures under the suspicion of more common (and serious) causes such as TB or lung malignancies. Only less than $10 \%$ of the subjects were apparently asymptomatic, although it should be recognized the low probability of a pauci- or asymptomatic case to be identified and described in the literature. Of particular note, a relatively large group of patients had Disseminated Histoplasmosis (DH), confirming that this condition, although typical of immunosuppressed individuals, may also occur in immunocompetent subjects. Moreover, given the long course of the infection, an unrecognized/untreated case of histoplasmosis may reactivate later in life, under immune suppressant conditions. Are there any definite risk factors for $\mathrm{DH}$ in immunocompetent individuals? We found several associations between disseminated histoplasmosis and other covariates at univariate analysis (Table 2). However, these results appear to be very inconsistent and were not confirmed by multivariate analysis and therefore no conclusion can be drawn from them. At multivariate analysis, the only risk factors resulting significant were exposition to cave-bats and outdoor activities. We found no association between the attack rate and the different endemic areas, hence we cannot suppose that there is a difference in the aggressiveness of the different strains throughout the world. In contrast with this conclusion, some authors [86-90] supposed that different and 


\section{Symptoms}

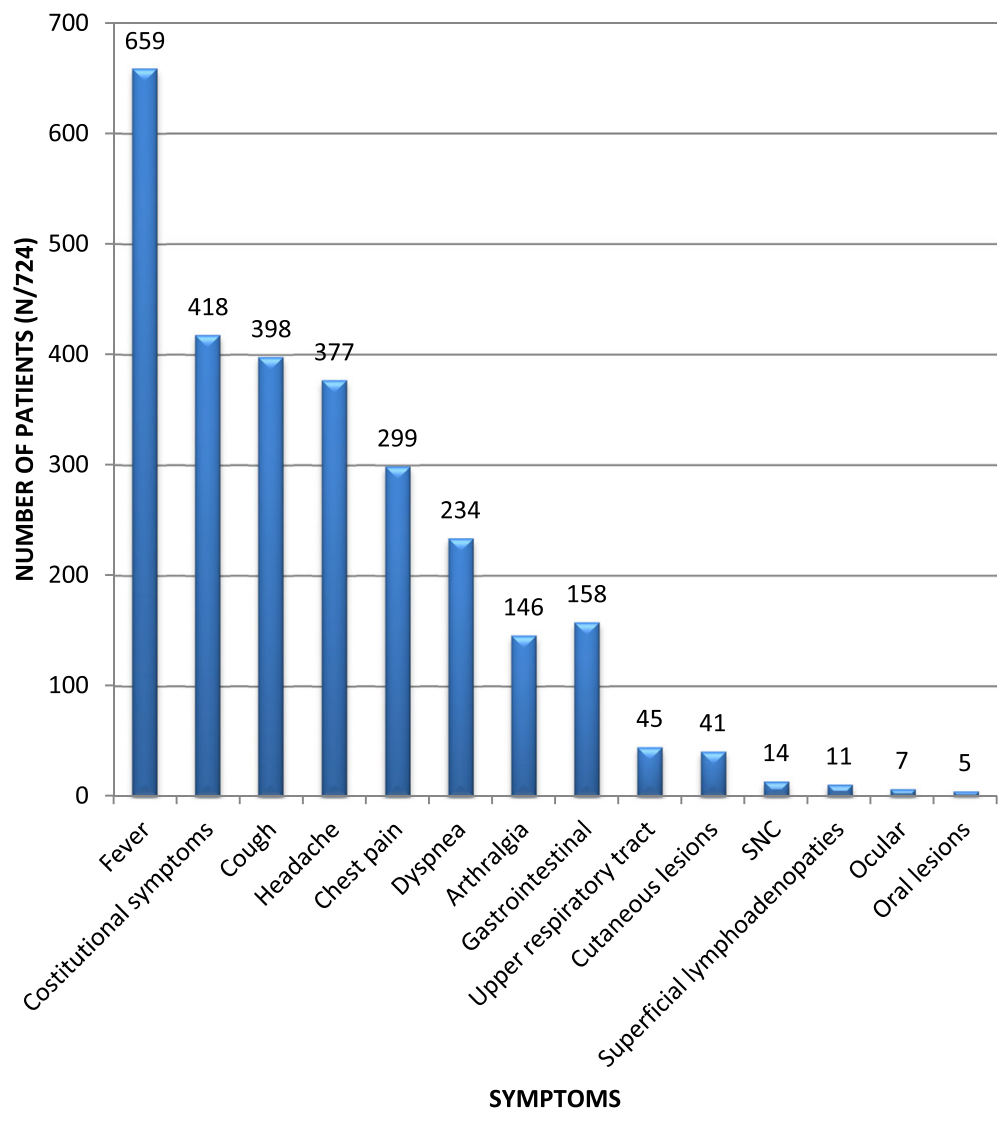

Fig. 4 Frequency of symptoms reported

specific Histoplasma capsulatum genotypes could be responsible of the variability of clinical manifestations, in particular some "aggressive" strains might cause a more severe presentation. It is to be mentioned that our result is based on 47 groups only, and the observation of more groups in endemic areas would possibly provide a more robust conclusion. Seventy-eight per cent of reported cases acquired the infection in the American continent, and $57,2 \%$ in Central-South America only; these areas should be therefore considered "highly" endemic, although the disease can be seen globally.

From the clinical point of view, when should histoplasmosis be suspected in a returning traveler? Histoplasmosis can be misdiagnosed, not only because of the common low awareness of health-care workers but especially because the clinical picture is mainly characterized by fever (more than $90 \%$ of reported cases) associated to respiratory symptoms in around half of cases. More than $80 \%$ of patients have a pulmonary form. Therefore, histoplasmosis should be suspected in all travelers presenting with fever/systemic symptoms or community-acquired pneumonia. History should be carefully evaluated, in particular exposure to cave/bats or simply outdoor activities in endemic areas (particularly the American continent) and being part of a cluster of similar illness. Although the most part of cases in our review had also abnormal laboratory exams (two thirds of cases had increased inflammatory markers and half increased transaminases) this is an aspecific finding, especially in pneumonia/febrile systemic illness. However, Histoplasma serology is positive in two third of cases. According to Wheat [4], Histoplasma galactomannan antigen can be positive in $80-95 \%$ of cases in the acute pulmonary and disseminated form and could strongly contribute to diagnosis when available.

Finally, radiologic findings can be of help to distinguish histoplasmosis from bacterial/viral pneumonia in immunocompetent travelers; in our review, $80 \%$ of retrieved cases with this information showed pulmonary nodules (typically multiple peripheric nodules with halo sign at computed tomography). Our study has some limitations. First, we excluded some papers by language, in particular those written in Chinese and Dutch, and this could have led to the loss of some cases. We did not find a standardized definition of acute histoplasmosis in the immunocompetent subject in the scientific literature, hence we adapted the definition and the inclusion 
Table 3 Crude and adjusted ORs for the Association Between Patient's Characteristics and the Risk of Disseminated Histoplasmosis (DH) among all Patients Recorded in Selected Studies

\begin{tabular}{|c|c|c|c|c|}
\hline \multirow[t]{2}{*}{ Covariate $^{a}$} & \multirow{2}{*}{$\begin{array}{l}\text { DH (\%) Present } \\
56 / 573(9.7)\end{array}$} & non-DH Absent & \multirow[t]{2}{*}{ Crude OR } & \multirow{2}{*}{$\begin{array}{l}\text { Adjusted OR }{ }^{b} \\
(95 \% \mathrm{Cl})^{\mathrm{b}}\end{array}$} \\
\hline & & $517 / 573(90.2)$ & & \\
\hline Age & $29(22-31)$ & $31(23-45)$ & $1.03(1.01-1.05)$ & $1.03(0.99-1.07)$ \\
\hline Male & $68.7 \%$ & $63.4 \%$ & $1.16(0.66-2.14)$ & $1.13(0.37-3.43)$ \\
\hline \multicolumn{5}{|c|}{ Continent of destination, N (\%) } \\
\hline USA-Canada & $3.8 \%$ & $22.5 \%$ & 1.00 [Reference] & 1.00 [Reference] \\
\hline Central America & $58.5 \%$ & $26.9 \%$ & $13.01(3.82-81.43)$ & $1.28(0.21-7.67)$ \\
\hline South America & $30.2 \%$ & $27.1 \%$ & $6.66(1.84-42.72)$ & $1.61(0.27-9.57)$ \\
\hline Africa & $5.7 \%$ & $18.0 \%$ & $1.87(0.30-14.45)$ & $2.38(0.28-20.71)$ \\
\hline Others $^{c}$ & $1.9 \%$ & $5.5 \%$ & $2.05(0.09-22.18)$ & $0.33(0.02-4.74)$ \\
\hline \multicolumn{5}{|l|}{ Activity $^{\mathrm{d}}, \mathrm{N}(\%)$} \\
\hline Caves/Bat guano & $64.2 \%$ & $60.5 \%$ & $1.18(0.66-2.11)$ & $34.20(5.29-220.93)$ \\
\hline Outdoor activities & $42.8 \%$ & $33.7 \%$ & $1.48(0.84-2.57)$ & $4.61(1.09-19.56)$ \\
\hline Bird_chickens & $10.7 \%$ & $19.1 \%$ & $0.50(0.19-1.13)$ & $7.19(0.84-61.57)$ \\
\hline Inhalation_soil & $37.5 \%$ & $16.0 \%$ & $3.13(1.72-5.62)$ & $2.78(0.94-8.29)$ \\
\hline
\end{tabular}

Abbreviations, $O R$ odds ratio, $\mathrm{Cl}$ confidence interval

${ }^{\text {a }}$ Relative frequencies are here presented, and the median (interquartile range) is reported for age

${ }^{b}$ Adjusted for every other covariate through a multivariate logistic regression model

c Includes: Asia, Oceania

d Each activity was entered in the model as a single dummy variable, as most people did more than one activity during the trip. Caves/Bat's guano: contact with bats excreta and/or visit to a cave. Outdoor activities: forest excursions/trekking, camping or other activities not involving visit to caves or direct contact with bats' excreta. Bird_chickens: activities involving contact with chickens or other birds. Inhalation_soil: all activities involving building construction, renovation, excavation (biologists, building contractors, construction workers, soldiers, volunteers involved in churches or other buildings renovation)

criteria on the basis of the works of prominent authors, namely Wheat and EORTC/MSG experts [4] [13, 14]. Most of all, it was difficult to retrieve accurate information about the outcome, that was not always described in details. However, with the limitation of a various and not ever reported follow-up, more than $99 \%$ of subjects recovered or had a consistent clinical and radiological improvement.

Inconsistency in the analysis of risk factors for Disseminated Histoplasmosis is most probably due to the low quality of the gathered data, that is probably explained by the vast heterogeneity of the sources across the years of publication and the differences in the statistics reported. We tried to implement different logistic models to verify which one could have a better goodness of fit, but unsuccessfully. We also tried data imputation to improve the completeness of the dataset. Namely, we tried to complete data on age and sex through a Multivariate Imputation by Chained Equations (MICE) [88] with the predictive mean matching method, in order to maintain the original distribution of age and sex of the incomplete data. Unfortunately, this did not have an impact on the quality of results. However, the exposure to cave-bats and outdoor activities resulted significant in the majority of models we fitted, so we assume that there is an association with those variables. We finally decided to report results from the best model in terms of goodness of fit.

\section{Conclusions}

Presumably, cases of histoplasmosis in immunocompetent travelers are largely misdiagnosed and under-reported, because these subjects are usually asymptomatic or present mild symptoms. However, it is important to consider this infection in the differential diagnosis in case of epidemiological risk of exposure, also in light of possible (though rare) progression of the disease also in immunocompetent individuals. In addition, the risk of reactivation of the infection in case of subsequent acquired immunosuppression is present, even after a long time [91]. Studies are needed to understand whether antifungal therapy prescribed for the acute, mild form of the disease could prevent further reactivations. Finally, a high index of suspicion might avoid unnecessary hospitalization and invasive procedures.

\section{Additional files}

Additional file 1: Excel sheet including all the demographical, clinical, radiological and treatment characteristics registered for each case. A row of the sheet $=$ a case. $($ XLSX $412 \mathrm{~kb})$

Additional file 2: COI disclosure of each Author. (DOCX 90 kb)

\section{Acknowledgements}

N.A.

\section{Funding}

This work did not receive any specific funding. 


\section{Availability of data and materials}

The data base created for the collection of cases and for the statistical analysis is available as supplementary material.

\section{Authors' contributions}

SS, AA independently screened the papers. When the opinion regarding the inclusion of a paper was discordant, a consensus was reached through discussion with a third reviewer, FG. MG and GG conducted the statistical analysis. DB, SS and AA wrote the manuscript. FB and ZB made the final revision of the manuscript. All Authors read and approved the final version of the manuscript.

\section{Ethics approval and consent to participate}

All the 21 patients seen at CTD gave their informed (verbal) consent for the publication of anonymous data.

We submitted a study protocol for the retrospective review of CTD cases to the Ethics Committee (Comitato Etico per la sperimentazione clinica delle Province di Verona e Rovigo), and received a waiver of consent (protocol 20,901 of 26 April 2017), since patients' data were de-identified.

\section{Consent for publication}

N.A.

\section{Competing interests}

The authors declare that they have no competing interests (Additional file 2).

\section{Publisher's Note}

Springer Nature remains neutral with regard to jurisdictional claims in published maps and institutional affiliations.

\section{Author details}

${ }^{1}$ Centro per le Malattie Tropicali, IRCCS Istituto di Ricovero e Cura a Carattere Scientifico, Ospedale Sacro Cuore - Don Calabria, Via Don Sempreboni 5, 37024 Verona, Negrar, Italy. ${ }^{2}$ SOD Malattie Infettive emergenti e degli immunodepressi, Azienda Ospedaliero Universitaria, Ospedali Riuniti di Ancona, Via Conca Torrette, Ancona, Italy. ${ }^{3}$ Computer Sciences, Dipartimento di Economia Aziendale, Università degli Studi di Verona, Strada le Grazie, Verona, Italy. ${ }^{4}$ Sezione di Malattie Infettive e Tropicali, Dipartimento di Diagnostica e Sanità Pubblica, Università di Verona, Strada le Grazie, Verona, Italy. ${ }^{5}$ Clinica Malattie Infettive, Dipartimento di Scienze Biomediche e Sanità Pubblica, Università Politecnica delle Marche, Azienda Ospedaliera Umberto I - Via Conca Torrette, Ancona, Italy.

Received: 13 October 2017 Accepted: 31 October 2018

Published online: 18 December 2018

\section{References}

1. Bahr NC, Antinori S, Wheat LJ, Sarosi GA. Histoplasmosis infections worldwide: thinking outside of the Ohio River valley. Curr Trop Med Rep. 2015;2(2):70-80

2. Hubalek Z. Emerging human infectious diseases: anthroponoses, zoonoses, and sapronoses. Emerg Infect Dis. 2003;9(3):403-4.

3. Emmons CW, Klite PD, Baer GM, Hill WBJ. Isolation of Histoplasma capsulatum from bats in the United States. Am J Epidemiol. 1966;84(1):103-9.

4. Wheat L, Azar MM, Bahr NC, Spec A, Relich RF, Hage C. Histoplasmosis. Infect Dis Clin N Am. 2016:30(1):207-27.

5. Goodwin RA Jr, Snell JD Jr. The enlarging histoplasmoma. Concept of a tumor-like phenomenon encompassing the tuberculoma and coccidioidoma. Am Rev Respir Dis. 1969;100(1):1-12.

6. Wheat $L$, Freifeld AG, Kleiman MB, Baddley JW, McKinsey DS, Loyd JE, Kauffman CA. Infectious diseases Society of a: clinical practice guidelines for the management of patients with histoplasmosis: 2007 update by the Infectious Diseases Society of America. Clin Infect Dis. 2007;45(7):807-25.

7. Lingscheid T, von Heinz M, Klages B, Rickerts V, Tintelnot K, Gerhold M, Oestmann JW, Becker M, Temmesfeld-Wollbruck B, Suttorp N, et al. A diagnostic predicament: activated sarcoidosis or pulmonary histoplasmosis. A case report. Clin Respir J. 2017;11(3):374-7.

8. Labhardt N, Rickerts V, Popescu S, Neumayr A. TB or not TB--persistent cough, fever and night sweats in a 46-year-old traveler returning from South America. Travel Med Infect Dis. 2015;13(4):346-8.
9. Sonavane AD, Sonawane PB, Chandak SV, Rathi PM. Disseminated histoplasmosis with Haemophagocytic Lymphohistiocytosis in an immunocompetent host. J Clin Diagn Res. 2016;10(3):OD03-5.

10. Pereira GH, Lanzoni VP, Beirao EM, Timerman A, Melhem Mde S. Disseminated fungal infection with adrenal involvement: report of two Hiv negative Brazilian patients. Rev Inst Med Trop Sao Paulo. 2015;57(6): $527-30$.

11. Choi SJ, Choi HS, Chun JY, Kim CJ, Lee MJ, Kim M, Choe GY. Subacute progressive disseminated histoplasmosis in immunocompetent patient. Korean J Intern Med. 2016;31(5):999-1002.

12. Ganesan N, Sharma R, Phansalkar MD, Varghese R. Disseminated histoplasmosis in an immunocompetent patient diagnosed on bone marrow aspirate - a rare presentation from a non-endemic area. J Clin Diagn Res. 2015;9(12):ED07-8.

13. Wheat LJ. Histoplasmosis: a review for clinicians from non-endemic areas. Mycoses. 2006;49(4):274-82.

14. De Pauw B, Walsh TJ, Donnelly JP, Stevens DA, Edwards JE, Calandra T, Pappas PG, Maertens J, Lortholary O, Kauffman CA, et al. Revised definitions of invasive fungal disease from the European Organization for Research and Treatment of cancer/invasive fungal infections cooperative group and the National Institute of Allergy and Infectious Diseases mycoses study group (EORTC/MSG) consensus group. Clin Infect Dis. 2008;46(12):1813-21.

15. Team RDC. R: a language and environment for statistical computing. Vienna: The R Foundation for statistical Computing; 2011.

16. Centers for Disease. C: cave-associated histoplasmosis--Costa Rica. MMWR Morb Mortal Wkly Rep. 1988;37(20):312-3.

17. Alonso D, Munoz J, Letang E, Salvado E, Cuenca-Estrella M, Buitrago MJ, Torres JM, Gascon J. Imported acute histoplasmosis with rheumatologic manifestations in Spanish travelers. J Travel Med. 2007;14(5):338-42.

18. Ansart S, Pajot O, Grivois JP, Zeller V, Klement E, Perez L, Bossi P, Bricaire F, Caumes E. Pneumonia among travelers returning from abroad. J Travel Med. 2004;11(2):87-91.

19. Ardizzoni A, Baschieri MC, Manca L, Salvadori C, Marinacci G, Farina C, Viale P, Blasi E. The mycoarray as an aid for the diagnosis of an imported case of histoplasmosis in an Italian traveler returning from Brazil. J Travel Med. 2013; 20(5):336-9.

20. Berwanger I, Hasse J, Schaefer HE, Kappe R, Muller J. The solitary pulmonary histoplasmoma. Dtsch Med Wochenschr. 1992;117(33):1232-6.

21. Bitterman R, Oren I, Geffen Y, Sprecher H, Schwartz E, Neuberger A. Prolonged fever and splinter hemorrhages in an immunocompetent traveler with disseminated histoplasmosis. J Travel Med. 2013;20(1):57-9.

22. Bonnet $\mathrm{D}$, Balandraud $\mathrm{P}$, Lonjon $T$, Rey $\mathrm{P}$, Van de Walle JP, Cador $\mathrm{L}$, Jancovici R, Martet G, Branquet D, De Muizon H. Round pulmonary lesions after returning from French Guyana. Six cases of american pulmonary histoplasmosis. Med Trop (Mars). 1995;55(1):55-60.

23. Buitrago MJ, Bernal-Martinez L, Castelli MV, Rodriguez-Tudela JL, CuencaEstrella M. Histoplasmosis and paracoccidioidomycosis in a non-endemic area: a review of cases and diagnosis. J Travel Med. 2011;18(1):26-33.

24. Buxton JA, Dawar M, Wheat LJ, Black WA, Ames NG, Mugford M, Patrick DM. Outbreak of histoplasmosis in a school party that visited a cave in Belize: role of antigen testing in diagnosis. J Travel Med. 2002;9(1):48-50.

25. Catania J, Martin SS, Corey GR, Sexton DS. Diagnostic dilemma in a returning traveler with fever. Diagn Microbiol Infect Dis. 2013;77(1):85-6.

26. Clyti E, Aznar C, Couppie P, Sainte-Marie D, Lemoine C, Huerre M, Carme B, Pradinaud R. Disseminated histoplasmosis detected by lingual and tonsillar erosions in an immunocompetent patient. Ann Dermatol Venereol. 1999; 126(10):709-11.

27. Cottle LE, Gkrania-Klotsas E, Williams HJ, Brindle HE, Carmichael AJ, Fry G, Beeching NJ. A multinational outbreak of histoplasmosis following a biology field trip in the Ugandan rainforest. J Travel Med. 2013;20(2):83-7.

28. Craven SA, Benatar SR. Histoplasmosis in the Cape Province. A report of the second known outbreak. S Afr Med J. 1979;55(3):89-92.

29. Cuguilliere A, N'Guyen G, Maslin J, Lonjon T, Miltgen J, Bonnet D. Tumor form of histoplasmosis in subjects returning from French Guiana Treatment of an immunocompetent patient. Presse Med. 1997;26(36):1724.

30. de Vries PJ, Koolen MG, Mulder MM, Kortbeek LM. Acute pulmonary histoplasmosis from Ghana. Travel Med Infect Dis. 2006;4(5):286-9.

31. Dean G. Cave disease. Cent Afr J Med. 1957;3(3):79-81.

32. Ehrhardt J, Tintelnot $K$, Kremsner $P$, Frank M. Progressive malaise and joint pain after travel to Costa Rica. Dtsch Med Wochenschr. 2012;137(44):2260. 
33. Erkens K, Lademann M, Tintelnot K, Lafrenz M, Kaben U, Reisinger EC. Histoplasmosis group disease in bat researchers returning from Cuba. Dtsch Med Wochenschr. 2002;127(1-2):21-5.

34. Ohno H, Ogata Y, Suguro H, Yokota S, Watanabe A, Kamei K, Yamagoe S, Ishida-Okawara A, Kaneko Y, Horino A, et al. An outbreak of histoplasmosis among healthy young Japanese women after traveling to Southeast Asia. Intern Med. 2010;49(5):491-5

35. Morgan J, Cano MV, Feikin DR, Phelan M, Monroy OV, Morales PK, Carpenter J, Weltman A, Spitzer PG, Liu HH, et al. A large outbreak of histoplasmosis among American travelers associated with a hotel in Acapulco, Mexico, spring 2001. Am J Trop Med Hyg. 2003;69(6):663-9.

36. Farina C, Gnecchi F, Michetti G, Parma A, Cavanna C, Nasta P. Imported and autochthonous histoplasmosis in Bergamo province, northern Italy. Scand J Infect Dis. 2000;32(3):271-4.

37. Farina C, Rizzi M, Ricci L, Gabbi E, Caligaris S, Goglio A. Imported and autochthonous histoplasmosis in Italy: new cases and old problems. Rev Iberoam Micol. 2005;22(3):169-71

38. Flor A, Estivills D, Perez R, Ordeig J, Ramos F, Soler Bel J, Puig X. Acute pulmonary histoplasmosis in a Spanish traveller to Nicaragua: an imported disease case. Rev Iberoam Micol. 2003;20(1):24-8.

39. Fuortes $L$, Hayes T. An outbreak of acute histoplasmosis in a family. Am Fam Physician. 1988;37(5):128-32.

40. Garcia-Vazquez E, Velasco M, Gascon J, Corachan M, Mejias T, TorresRodriguez JM. Histoplasma capsulatum infection in a group of travelers to Guatemala. Enferm Infecc Microbiol Clin. 2005;23(5):274-6.

41. Gascon J, Torres JM, Luburich P, Ayuso JR, Xaubet A, Corachan M. Imported histoplasmosis in Spain. J Travel Med. 2000;7(2):89-91.

42. Gelfand M. Cave disease. A report of 3 cases from southern Rhodesia. Cent Afr J Med. 1962;8:461-6.

43. Guigay J, Natali F, Schill H, Fassa Y, Allard P. Radiological case: a peculiar ganglio-pulmonary involvement. Rev Pneumol Clin. 1990;46(6):277-9.

44. Gustafson TL, Kaufman L, Weeks R, Ajello L, Hutcheson RH Jr, Wiener SL, Lambe DW Jr, Sayvetz TA, Schaffner W. Outbreak of acute pulmonary histoplasmosis in members of a wagon train. Am J Med. 1981;71(5):759-65.

45. Handzel S, Jessamine AG. Imported histoplasmosis from Puerto Rico. Can J Public Health. 1975;66(5):393-5

46. Hay RJ. Mycoses imported from the West Indies. A report of three cases. Postgrad Med J. 1979;55(647):603-4.

47. Hirsch D, Leupold W, Rupprecht E. Pulmonary histoplasmoma after travel abroad. Pneumologie. 1996;50(3):242-4.

48. Hoenigl M, Schwetz I, Wurm R, Scheidl S, Olschewski H, Krause R. Pulmonary histoplasmosis in three Austrian travelers after a journey to Mexico. Infection. 2008;36(3):282-4.

49. Julg B, Elias J, Zahn A, Koppen S, Becker-Gaab C, Bogner JR. Bat-associated histoplasmosis can be transmitted at entrances of bat caves and not only inside the caves. J Travel Med. 2008;15(2):133-6.

50. Kajfasz P, Basiak W. Outbreak of pulmonary histoplasmosis involving a group of four polish travellers returning from Ecuador. Int Marit Health. 2012;63(1):59-62.

51. Locatelli Sanchez M, Mornex JF, Philit F, Chumbi Floresa W. Pulmonary nodules upon return from Costa Rica. Rev Mal Respir. 2011;28(7):938-9.

52. Kiessling, F, et al. Pulmonary nodules in a patient with high temperature. Acute pulmonary histoplasmosis. Radiologe. 2003;43(10):856-60.

53. Lottenberg R, Waldman RH, Ajello L, Hoff GL, Bigler W, Zellner SR. Pulmonary histoplasmosis associated with exploration of a bat cave. Am J Epidemiol. 1979;110(2):156-61.

54. Lyon GM, Bravo AV, Espino A, Lindsley MD, Gutierrez RE, Rodriguez I, Corella A, Carrillo F, McNeil MM, Warnock DW, et al. Histoplasmosis associated with exploring a bat-inhabited cave in Costa Rica, 1998-1999. Am J Trop Med Hyg. 2004;70(4):438-42

55. Manolakos JJ, Cooray M, Patel A, Haider S. Bats, fever and adenopathy what is the link? Can J Infect Dis Med Microbiol. 2013:24(1):35-7.

56. Margery J, Bonnichon A, Jeanbourquin D, Saint-Blancard P, Sane M, Dot JM, Grassin F, Vaylet F, L'Her P, Guigay J. Pseudotumoral presentation of American pulmonary histioplasmosis. Rev Pneumol Clin. 2005;61 (6):365-8.

57. Mongeal E, Balduin MT, Voloch A, Souquet PJ, Pinet S, Piens MA, Guibert B, Bernard JP. Pulmonary histoplasmosis due to Histoplasma capsulatum a case. Rev Mal Respir. 1991:8(5):495-7.

58. Muller-Stover I, Tintelnot K, Richter J, Haussinger D. A 55-year-old woman with cough, fever, swelling of joints, and exanthema after a trip to Ecuador. Internist (Berl). 2014;55(8):976-80.
59. Murray JF, Lurie HI, Kaye J, Komins C, Borok R, Way M. Benign pulmonary histoplasmosis (cave disease) in South Africa. S Afr Med J. 1957;31(11):245-53.

60. Nasta P, Donisi A, Cattane A, Chiodera A, Casari S. Acute histoplasmosis in spelunkers returning from Mato Grosso, Peru. J Travel Med. 1997;4(4):176-8.

61. Noel M, Levenes H, Duval P, Barbe C, Ramognino P, Verhaegen F. Epidemic of pulmonary histoplasmosis after visiting a cave in New Caledonia. Sante. 1995;5(4):219-25.

62. Norman FF, Martin-Davila P, Fortun J, Dronda F, Quereda C, Sanchez-Sousa A, Lopez-Velez R. Imported histoplasmosis: two distinct profiles in travelers and immigrants. J Travel Med. 2009;16(4):258-62.

63. Olalla J, Ramon Costa J, Rioperez E, Yubero B. Acute pulmonary histoplasmosis after a journey to Peru. Med Clin (Barc). 2001;116(17):679.

64. Rocha-Silva F, Figueiredo SM, Silveira TT, Assuncao CB, Campolina SS, PenaBarbosa JP, Rotondo A, Caligiorne RB. Histoplasmosis outbreak in Tamboril cave-Minas Gerais state. Brazil Med Mycol Case Rep. 2014;4:1-4.

65. Sacks JJ, Ajello L, Crockett LK. An outbreak and review of cave-associated histoplasmosis capsulati. J Med Vet Mycol. 1986;24(4):313-25.

66. Salhab KF, Baram D, Bilfinger TV. Growing PET positive nodule in a patient with histoplasmosis: case report. J Cardiothorac Surg. 2006;1:23.

67. Salomon J, Flament Saillour M, De Truchis P, Bougnoux ME, Dromer F, Dupont B. de saint-Hardouin G, Perronne C: an outbreak of acute pulmonary histoplasmosis in members of a trekking trip in Martinique. French West Indies J Travel Med. 2003;10(2):87-93.

68. Schramm N, Bogner J, Broeckel N, Becker-Gaab C. Biologist with cephalalgia and intermittend fever. Radiologe. 2009;49(1):68-71.

69. Segel MJ, Rozenman J, Lindsley MD, Lachish T, Berkman N, Neuberger A, Schwartz E. Histoplasmosis in Israeli travelers. Am J Trop Med Hyg. 2015; 92(6):1168-72

70. Senechal A, Ferry T, Boibieux A, Brion JP, Epaulard O, Chidiac C, Peyramond D. Imported pulmonary histoplasmosis in three French cavers after a trip to Cuba. J Travel Med. 2012;19(1):64-5.

71. Shimamoto A, Takao M, Shomura S, Tarukawa T, Shimpo H. Pulmonary histoplasmosis as an example of imported mycoses in Japan. Gen Thorac Cardiovasc Surg. 2007;55(8):335-8.

72. Tinelli M, Michelone G, Cavanna C. Recurrent Histoplasma capsulatum pneumonia: a case report. Microbiologica. 1992;15(1):89-93.

73. Torres-Rodriguez JM, Ribas-Forcadell E, Gascon J, Lopez-Jodra O, Espasa M. Diagnostic usefulness of the intradermal test with histoplamin in nonendemic areas of histoplasmosis. Rev Iberoam Micol. 2000;17(3):97-101.

74. Valdez H, Salata RA. Bat-associated histoplasmosis in returning travelers: case presentation and description of a cluster. J Travel Med. 1999;6(4):258-60.

75. Centers for Disease C. Prevention: outbreak of histoplasmosis among travelers returning from El Salvador--Pennsylvania and Virginia, 2008. MMWR Morb Mortal Wkly Rep. 2008;57(50):1349-53.

76. Weinberg M, Weeks J, Lance-Parker S, Traeger M, Wiersma S, Phan Q, Dennison D, MacDonald P, Lindsley M, Guarner J, et al. Severe histoplasmosis in travelers to Nicaragua. Emerg Infect Dis. 2003;9(10):1322-5.

77. Wolff M. Outbreak of acute histoplasmosis in Chilean travelers to the ecuadorian jungle: an example of geographic medicine. Rev Med Chil. 1999;127(11):1359-64.

78. Wyborney VJ, Walch $\mathrm{H}$, Loeffler AB, Wiita RM. Histoplasmosis. A review of three cases studied in San Diego County. Calif Med. 1966;105(4):265-70.

79. Salama H, Wheat $L$, Sobel JD, Srivastava R, Richer SM, Rongkavilit C. Coughing spells in a Michiganian girl after a trip to southwest US. Pediatr Infect Dis J. 2015:34(5):546 553-544

80. Zollner MS, Rezende KM, Birman S, Elias CP, Arisawa EA, Santos MA. Clinical and evolutionary characteristics of four patients with pulmonary histoplasmosis reported in the Paraiba Paulista Valley region. Rev Soc Bras Med Trop. 2010;43(5):599-601.

81. Corti MNR, Palmieri O. Brote de histoplasmosis de primoinfeccion en cinco hermanas. Revista de patologia Tropical. 2012;41(1):8.

82. Cabello HNM, Meneses M, Peralta M, Larraguibel C. Histoplasmosis pulmonar. Revista Chilena de Infectologia. 2002;19(1):6.

83. Panizo M, Dolande M, Reviakina V, Maldonado B. Histoplasmosis pulmonar asociada con visita a cuevas: Descripción de un brote epidémico y revisión de la literatura, vol. 21; 2001

84. Castaneda Hernandez AMGG, Miguel A, Guzman U. Histoplasmosis epidemica. Aspectos clinicos y serologicos Biomedica. 1981;1(1):7.

85. Liu JW, Huang TC, Lu YC, Liu HT, Li CC, Wu JJ, Lin JW, Chen WJ. Acute disseminated histoplasmosis complicated with hypercalcaemia. J Inf Secur. 1999;39(1):88-90. 
86. Karimi K, Wheat L, Connolly P, Cloud G, Hajjeh R, Wheat E, Alves K, Lacaz $\mathrm{Cd}$ Cda S, Keath E. Differences in histoplasmosis in patients with acquired immunodeficiency syndrome in the United States and Brazil. J Infect Dis. 2002:186(11):1655-60.

87. Machado AA, Coelho IC, Roselino AM, Trad ES, Figueiredo JF, Martinez R, de Costa JC. Histoplasmosis in individuals with acquired immunodeficiency syndrome (AIDS): report of six cases with cutaneous-mucosal involvement. Mycopathologia. 1991;115(1):13-8.

88. Goldani LZ, Aquino VR, Lunardi LW, Cunha VS, Santos RP. Two specific strains of Histoplasma capsulatum causing mucocutaneous manifestations of histoplasmosis: preliminary analysis of a frequent manifestation of histoplasmosis in southern Brazil. Mycopathologia. 2009;167(4):181-6.

89. Durkin MM, Connolly PA, Karimi K, Wheat E, Schnizlein-Bick C, Allen SD, Alves K, Tewari RP, Keath E. Pathogenic differences between north American and Latin American strains of Histoplasma capsulatum var. capsulatum in experimentally infected mice. J Clin Microbiol. 2004;42(9): 4370-3.

90. Negroni R. Histoplasmosis in Latin America. Biomedica. 2011;31(3):304.

91. Gajurel K, Dhakal R, Deresinski S. Histoplasmosis in transplant recipients. Clin Transpl. 2017;31(10):e13087.

Ready to submit your research? Choose BMC and benefit from:

- fast, convenient online submission

- thorough peer review by experienced researchers in your field

- rapid publication on acceptance

- support for research data, including large and complex data types

- gold Open Access which fosters wider collaboration and increased citations

- maximum visibility for your research: over $100 \mathrm{M}$ website views per year

At BMC, research is always in progress.

Learn more biomedcentral.com/submissions 